\title{
GMDH-type Neural Networks for Predicting Financial Time Series: A Study of Informational Efficiency of Stock Markets
}

\section{Jakaite}

University of Bedfordshire - Luton Campus: University of Bedfordshire

\section{Ciemny}

EY-Parthenon

\section{S Selitskiy}

University of Bedfordshire - Luton Campus: University of Bedfordshire

Vitaly Schetinin ( $\square$ vitaly.schetinin@beds.ac.uk)

University of Bedfordshire https://orcid.org/0000-0003-1826-0153

\section{Research Article}

Keywords: Financial Time Series, Prediction, Efficient Market Hypothesis, Deep Learning

Posted Date: November 9th, 2021

DOI: https://doi.org/10.21203/rs.3.rs-1017105/v1

License: (c) (i) This work is licensed under a Creative Commons Attribution 4.0 International License. Read Full License 


\title{
GMDH-type Neural Networks for Predicting Financial Time Series: A Study of Informational Efficiency of Stock Markets
}

\author{
L. Jakaite • M. Ciemny · S. Selitskiy • \\ V. Schetinin*
}

Received: date / Accepted: date

\begin{abstract}
A theory of Efficient Market Hypothesis (EMH) has been introduced by Fama to analyse financial markets. In particular the EMH theory has been proven in real cases under different conditions, including financial crises and frauds. The EMH assumes to examine the prediction accuracy of models designed on retrospective data. Such prediction models could be designed in different ways that motivated us to explore Machine Learning (ML) methods known for building models providing a high prediction performance. In this study we propose a "deep" learning method for building high-performance prediction models. The proposed method is based on the Group Method of Data Handling (GMDH) that is the deep learning paradigm capable of building multilayer neural-network models of a near-optimal complexity on given data. We show that the developed GMDH-type neural network has outperformed the models built by the conventional ML methods on the Warsaw Stock Exchange data. It is important that the complexity of the designed GMDH-type neuralnetworks is defined by the number of layers and connections between neurons. The performances of models were compared in terms of the prediction errors. We report a significantly smaller prediction error of the proposed method than that of the conventional autoregressive and "shallow" neural-network models. This finally allows us to conclude that traders will be advantaged by the proposed method.
\end{abstract}

Keywords Financial Time Series · Prediction - Efficient Market Hypothesis · Deep Learning

\author{
*V. Schetinin \\ Department of Computer Science and Technology \\ University of Bedfordshire \\ Park Square, Luton, UK \\ E-mail: vitaly.schetinin@beds.ac.uk
}




\section{Introduction}

The Efficient Market Hypothesis (EMH) has been considered as a disputable theory since Fama's publications $[7,8]$. There are still many open questions on how this theory can be used for reliable analysis of the financial markets, essentially during financial crises and in the cases of frauds, see e.g. [19,6,11, 10]. The market anomalies are very intriguing events which may affect the efficiency of the stock market. There are many methods proposed to verify the EMH, however none of them have been proved to be reliable. The promising approach is based on Machine Learning (ML) methods capable of building forecasting models which can be reliably used for testing the EMH, see e.g. $[32,27,21]$.

Investors want to reliably predict the future behaviour of the stock market in order to minimise risks. There are many factors which affect the market behaviour. The theory of EMH has been studied and extended by Fama, following an early work assuming that the prices fully reflect all information available on the stock market. The study has particularly focused on the cases when the stock market prices cannot be reliably predicted. Based on these findings the stock market efficiency was defined as strong, semi-strong, and weak. Further study has been undertaken in [2,10,34].

The strong form defines the cases when financial information is available for each trader at the same time and there is no a person who can take an unfair advantage beforehand and use the information for own profit. Everyone has the full information on the stock market so that an abnormal profit cannot be achieved, and as a result the market is defined as fully efficient. There are no patterns in the retrospective data which traders can use for making a profit in the future. This means that the future prices cannot be predicted, see e.g. $[11,10]$.

According to Fama's theory, the future prices are entirely dependent on the information available for traders. When new information is released to the market, the stock prices are adjusted to an equilibrium point at which an abnormal profit cannot be achieved.

Conventional methods such as Autoregressive (AR) modelling have been applied to build models for stock price predictions [15,33]. Artificial NeuralNetwork (ANN) models have been applied to predict the stock market indexes. The use of ANNs requires defining an error function, a neural-network structure, as well as a gradient method capable to iteratively reduce the given error function, see e.g. $[1,25,14,3,23,5]$.

Multilayer neural networks are efficiently built by Deep Learning, as shown in [31]. Following $[12,9,18]$, the multilayer neural-network models could be grown by the Group Method of Data Handling (GMDH) using a neuron activation function defined by a short-term polynomial. The GMDH is capable of generating new layers capable of predicting new data most accurately. In each layer the GMDH generates new neurons to be fitted to the training data. A given number of the best fitted neurons are selected to the next layer. The number of layers increases whilst the special, so-called exterior, criteria have 
a tendency to decrease. The use of such selection criteria enables the GMDH to efficiently avoid the network over-fitting, as described e.g. in $[26,24,20]$. Different self-organising strategies were recently reviewed in [17].

In particular ML methods have been efficiently used to solve problems such as detection of abnormal patterns and evaluation of brain development in newborn electroencephalograms [30]. The reliable results have been achieved in prediction of trauma survival [28], air-traffic collision avoidance [29], as well as in detection of bone pathologies [13].

In this paper we describe our findings and insights into the problem of using the theory of Informational Efficiency of stock markets within the Machine Learning framework. We provide details of the GMDH-framework proposed for building neural network models of a near-optimal connectivity and then describe our experiments on testing the weak form of Informational Efficiency on the Warsaw Stock Exchange during 2007-2009. In our study we compare the modelling techniques based on the AR modelling, ANN, and GMDH-type neural-network models. Finally we make conclusions on the results obtained in our comparative experiments.

\section{Methods \& Data}

2.1 Autoregressive models

According to $[33,1,25]$, an AR model aims to explain a variable $x_{k+1}$ at the time $k+1$ by using the lagged variables $x_{k}, x_{k-1}, \ldots, x_{k-M+1}$ as follows:

$$
x_{k+1}=\alpha_{0}+\alpha_{1} x_{k}+\alpha_{2} x_{k-1}+\cdots+\alpha_{M} x_{k-M+1}+e_{k+1},
$$

where $\alpha=\left(\alpha_{0}, \alpha_{1}, \ldots, \alpha_{M}\right)$ is a vector of the adjustable parameters of an AR model, $M$ is the order of the AR model, and $e_{k+1}$ is the prediction error at the time $k+1$.

The above prediction error is minimised by fitting the AR parameters $\alpha$ to the retrospective data. In particular the error could be minimised by using a sampler based on Markov chains. The use of such samplers can additionally provide a prediction with the reliable estimates of predictive posterior density distribution, which is critically important for making risk-aware decisions in the presence of uncertainties, see e.g. [29].

\subsection{Feed-forward Neural Networks}

In our comparative experiments we used a conventional ANN consisting of one hidden layer and one output neuron. The neurons of hidden layer read the $M$ lagged samples of a time series $\left\{x_{k}, \ldots, x_{k-M+1}\right\}$, where $M$ is the number of lags, which is required to be experimentally found to minimise the prediction error, see e.g. $[3,23,5]$. 
A linear activation function for the neurons in the hidden and output layers was used. The backpropagation gradient algorithm has been used with an early stopping rule to avoid the over-fitting problem. The use of momentum has increased the ability of finding the global minimum of the error function. The initial neural-network weights were set at random. The number of epochs (or iterations) used for training the neural network has been set to 1000 .

\subsection{Group Method of Data Handling}

The analysis of limitations of the existing ANN solutions gives us new insights into the problem of predicting the financial time series. In this section we describe the key steps of the proposed GMDH-type neural-network framework.

The key idea of the GMDH is to use Gabor-Kolmogorov (GK) polynomials for learning a multivariate prediction model from data. In the general form, the GK polynomials are written as follows:

$y\left(x_{1}, \ldots, x_{m}\right)=w_{0}+\sum_{i=1}^{m} w_{i} x_{i}+\sum_{i=1}^{m} \sum_{j=1}^{m} w_{i j} x_{i} x_{j}+\sum_{i=1}^{m} \sum_{j=1}^{m} \sum_{k=1}^{m} w_{i j k} x_{i} x_{j} x_{k}+\ldots$

Here $m$ is the number of the input variables $\left\{x_{1}, x_{2}, \ldots, x_{m}\right\}$ or lags used for prediction. In practice the number $m$ is unknown and users need to run multiple experiments to find a model which provides the minimal prediction error. The coefficients $w_{0}, w_{i}, w_{i j}, w_{i j k}, \ldots$ are fitted to the data as to minimise the error between the model's output and actual values.

The above polynomial decomposition (Eq. 2) employs short-term polynomials $g\left(x_{i}\right), g\left(x_{i}, x_{j}\right), g\left(x_{i}, x_{j}, x_{k}\right)$. The use of the 2-order polynomials $g\left(x_{i}, x_{j}\right)$ makes the learning computationally and statistically feasible, as shown in [12, $9,18]$.

For example, using the GK polynomial decomposition, a function $y=$ $f\left(x_{1}, x_{2}, x_{3}\right)$ can be composed of the following 2-argument polynomials and the bias $g_{0}$ :

$$
y\left(x_{1}, x_{2}, x_{3}\right)=g_{0}+g_{1}\left(x_{1}, x_{2}\right)+g_{2}\left(x_{1}, x_{3}\right)+g_{3}\left(x_{2}, x_{3}\right) .
$$

In general, such a decomposition significantly reduces the number of model's adjustable parameters as well as the number of data samples required to achieve the computational and statistical feasibility.

It is important to note that the GMDH is efficiently implemented when the collinearity of variables generated in multiple layers is avoided. The proposed GMDH algorithm is terminated at a layer $r^{*}$ when the number of independent outputs of neurons becomes below a given threshold.

Using a given reference polynomial $g(\mathbf{x} ; \mathbf{w})$, the algorithm generates a feature vector $\mathbf{x}=\left(x_{i_{1}}, x_{i_{2}}\right)$ for $K$ neurons at the 1st layer $r=1$, where $i_{1} \neq i_{2}, i_{1}=1, \ldots, m$, and $K=m(m-1) / 2$, is the number of pairwise combinations for the given $m$ inputs. The outputs of neurons at the $r$ th layer, $\mathbf{y}_{i}$, are written as: 


$$
\mathbf{y}_{i}^{(r)}=g\left(x_{i_{1}}, x_{i_{2}} ; \hat{\mathbf{w}}_{i}^{(r)}\right), i=1, \ldots, K
$$

Given a set $\mathcal{A}$ of the training instances, the coefficient vectors $\hat{\mathbf{w}}^{(r)}$ are fitted to the data $[\mathbf{X}(\mathcal{A}) \mid \stackrel{\circ}{\mathbf{y}}(\mathcal{A})]$ by solving the normal equations. Their solutions are numerically stable as the number of instances included in the set $\mathcal{A}$ is larger than the number of the variables $m$ and the columns $i_{1}, i_{2}$ of the matrix $\mathbf{X}\left(\mathcal{A},\left[i_{1}, i_{2}\right]\right)$ are not correlated, so that the estimates $\hat{\mathbf{w}}$ are :

$$
\hat{\mathbf{w}}^{(r)}=\left[\mathbf{1} \mathbf{X}\left(\mathcal{A},\left[i_{1}, i_{2}\right]\right)\right]^{-1} \mathbf{y}(\mathcal{A}),
$$

were 1 denotes the unit vector.

Having the outputs $\mathbf{y}_{i}$ on the entire data $\mathbf{X}$, the regularisation errors $\Delta$ can be calculated as follows:

$$
\Delta_{i}=\left\|\mathbf{y}_{i}-\stackrel{\circ}{\mathbf{y}}\right\|
$$

The errors are sorted into ascending order, and then the first $F$ neurons with lowest errors are selected for the next layer. The neurons with a correlation between their outputs exceeding a threshold are excluded from the selection $\mathbf{y}_{i_{1}}, \ldots, \mathbf{y}_{i_{F}}$. So the number of selected neurons after the decorrelation at the $r$ th layer can be $F_{r}: 1 \leq F_{r} \leq F$.

Neurons at the next layers $r+1$ are generated by applying the polynomials $g$ to an extended data matrix $\mathbf{Z}^{(r)}=\left[\mathbf{Y}^{(r)} \mid \mathbf{X}\right]$, which includes the outputs of the selected neurons $\mathbf{Y}^{(r)}=\left[\mathbf{y}_{i_{1}}, \ldots, \mathbf{y}_{i_{F_{r}}}\right]$, and the input data $\mathbf{X}$, so that the matrix $\mathbf{Z}^{(r)}$ contains $m_{r}=F_{r}+m$ columns.

Similarly, the coefficient vectors $\hat{\mathbf{w}}^{(r+1)}$ are estimated for the $i_{1}, i_{2}$ columns of the matrix $\mathbf{Z}^{(r)}$. The outputs of neurons $\mathbf{y}^{(r+1)}$ are then calculated as follows:

$$
\mathbf{y}_{i}^{(r+1)}=g\left(\mathbf{z}_{i_{1}}^{(r)}, \mathbf{z}_{i_{2}}^{(r)} ; \hat{\mathbf{w}}_{i}^{(r+1)}\right), i=1, \ldots, K_{r},
$$

where $i_{1} \neq i_{2} ; i_{1}=1, \ldots, m_{r}, i_{2}=1, \ldots, m_{r}$, and $K_{r}=m_{r}\left(m_{r}-1\right) / 2$ are the number of pairwise combinations for the $m_{r}$ columns.

Generating new layers as described above, the algorithm allows the network to grow while the number $F_{r}$ is larger than a given threshold. The pseudocode of the algorithm is outlined below.

The main steps of the proposed algorithm are represented by the Algorithm 1 . The network is initialised at line 2 for the given training data $[\mathbf{X} \mid \stackrel{\mathbf{y}}{]}]$ and the number of neurons $F$. Then at line 4 the Algorithm generates the $r=1$ layer. Then a new layer $r$ is generated at line 8 . The neurons generated at the $r$ th layer are selected at line 9 . The algorithm stops if the number of selected neurons, $F_{r}$, is fewer than a given threshold $F_{0}$.

Finally the trained neural network is represented by the parameters saved in the fields of the matrix Net. 
Fig. 1 Log returns of WIG index between 18/01/2007 and 30/08/2009
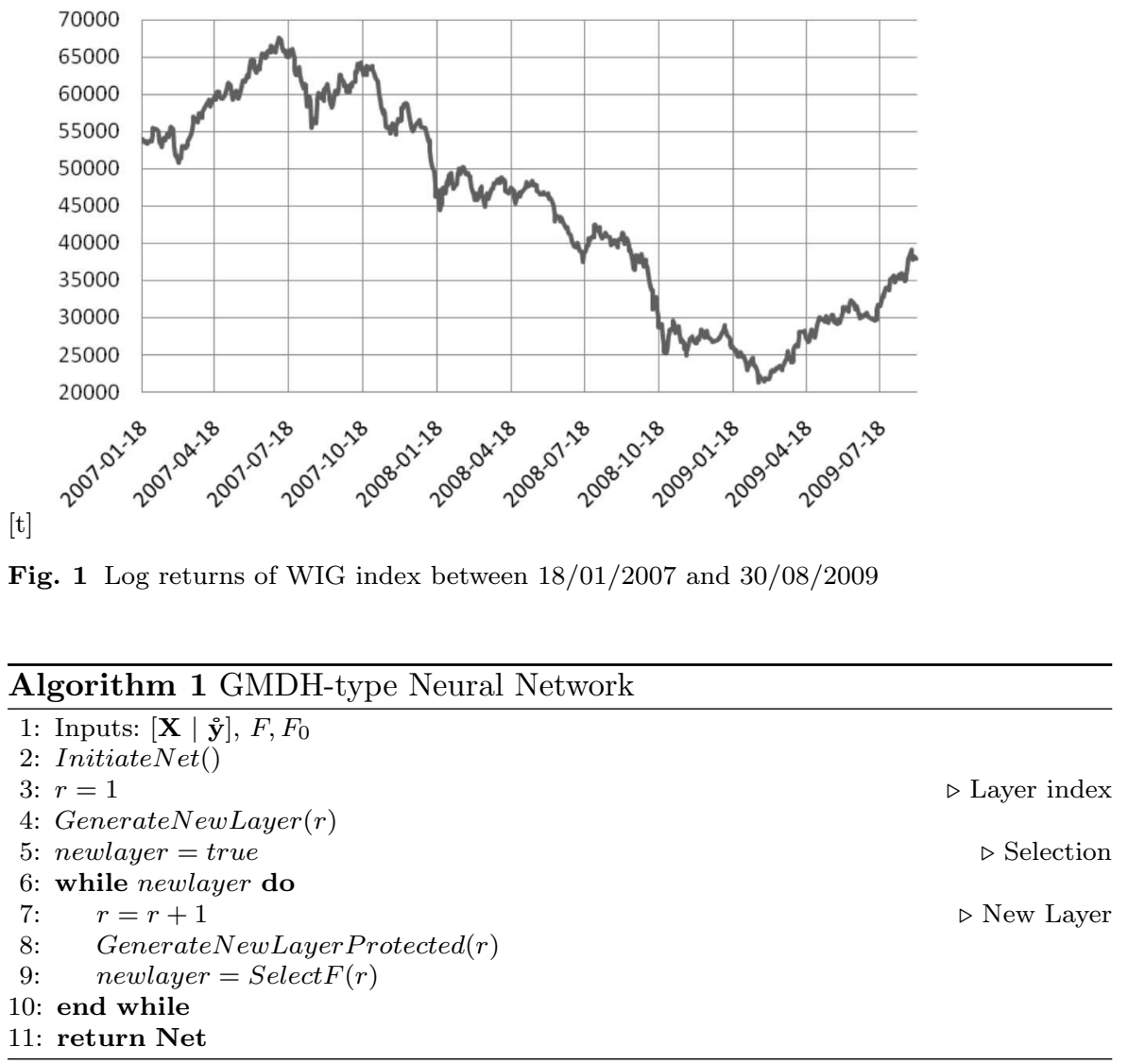

\subsection{Data}

In our study we aim to verify a weak form of Informational Efficiency of the stock market on the data from the Warsaw Stock Exchange during the crisis during 2007-2009. The data represent the daily rate of return on the main index WIG. The original data were transformed into a logarithmic rate as follows: $R_{t}=\ln \left(P_{t} / P_{t-1}\right)$, here $R_{t}$ is the logarithmic rate of return at time $t$, $P_{t}$ is the size in points of the WIG index at time $t$.

We used the data between 18/01/2009 and 30/08/2009, which represent 655 observations of the daily returns. Fig. 1 shows these data.

The data were divided into 13 subsets, each consisting of 50 observations used as a data set for building a prediction model. The remaining 5 observations were used as a validation set required to evaluate the prediction performance of the prediction model. Thus in our experiments we followed the work [4] which has suggested to use 5 observations (or one week listing period) for short-term predictions. 
Table 1 MAPE calculated for the AR, ANN, and GMDH models on the test data over 13 sessions

\begin{tabular}{llllll}
\hline$\#$ & Train period & Test period & AR & ANN & GMDH \\
1 & $07-01-18-07-03-28$ & $07-03-29-07-04-04$ & 0.97 & $\mathbf{0 . 0 8}$ & 0.18 \\
2 & $07-03-29-07-06-13$ & $07-06-14-07-06-20$ & 1.29 & $\mathbf{0 . 1 4}$ & 0.26 \\
3 & $07-06-14-07-08-23$ & $07-08-24-07-08-30$ & 4.09 & 1.11 & $\mathbf{0 . 4 2}$ \\
4 & $07-08-24-07-11-02$ & $07-11-05-07-11-09$ & 1.48 & $\mathbf{0 . 4 2}$ & 1.60 \\
5 & $07-11-05-08-01-18$ & $08-01-21-08-01-25$ & 1.83 & 2.51 & $\mathbf{0 . 7 9}$ \\
6 & $08-01-21-08-04-01$ & $08-04-02-08-04-08$ & 1.24 & $\mathbf{0 . 1 4}$ & 0.27 \\
7 & $08-04-02-08-06-13$ & $08-06-16-08-06-20$ & $\mathbf{1 . 0 1}$ & 2.88 & 1.65 \\
8 & $08-06-16-08-08-25$ & $08-08-26-08-09-01$ & 1.15 & $\mathbf{0 . 1 7}$ & 2.28 \\
9 & $08-08-26-08-11-03$ & $08-11-04-08-11-10$ & 1.16 & 0.74 & $\mathbf{0 . 2 6}$ \\
10 & $08-11-04-09-01-20$ & $09-01-21-09-01-27$ & $\mathbf{1 . 0 5}$ & 4.44 & 1.66 \\
11 & $09-01-21-09-03-31$ & $09-04-01-09-04-07$ & 1.16 & $\mathbf{0 . 1 6}$ & 0.39 \\
12 & $09-04-01-09-06-15$ & $09-06-16-09-06-22$ & 2.03 & $\mathbf{0 . 1 8}$ & 0.89 \\
13 & $09-06-16-09-08-24$ & $09-08-25-09-08-31$ & 1.09 & $\mathbf{0 . 1 5}$ & 0.20 \\
\hline
\end{tabular}

In order to verify the accuracy and usefulness of predictions made by different ML models, we use the Average Absolute Percentage Error (MAPE) defined as follows:

$$
M A P E=\frac{1}{n} \sum_{t=1}^{n}\left|\frac{A_{t}-F_{t}}{A_{t}}\right|,
$$

where $A_{t}$ and $F_{t}$ are the actual and predicted indexes, respectively, and $n$ is the number of observations.

The above splits of the data into 13 sessions allows us to perform 13-fold cross-validation to compare the prediction performance of different models. Each session includes 50 observations for building a prediction model and the remaining 5 observations are used for evaluation of prediction performance in terms of the MAPE defined by Eq. 8. The calculations were performed for each session, and then the average MAPE over all 13 sessions were used for comparison of the model's prediction performance.

\section{Results}

The results obtained in our experiments on the Warsaw Stock Exchange data are shown in Table 1 and Table 2. The performances of the AR, ANN, and proposed GMDH methods are represented by the MAPE values calculated on the test data set for all 13 sessions. This analysis allows us to assess each prediction model within the 13 -fold cross-validation.

Following the EMH theory, we will compare the MAPE calculated for each model with the random walk for which the $M A P E=1.0$. The results of our comparative study are as follows.

We can see that the prediction performance of the AR model is lowest as the average MAPE is 1.5. The performance of the ANN model achieved 1.0 
Table 2 Average MAPE over 13 sessions calculated for AR, ANN, and GMDH models

\begin{tabular}{llll}
\hline MAPE & AR & ANN & GMDH \\
Average & 1.50 & 1.01 & $\mathbf{0 . 8 3}$ \\
Standard deviation & 0.84 & 1.39 & $\mathbf{0 . 7 2}$ \\
Variance & 0.71 & 1.93 & $\mathbf{0 . 5 2}$ \\
\hline
\end{tabular}

which is the same as for the random walk. The best performance is provided by the GMDH-type model with the average MAPE equal to 0.83 .

It is also important to note that the GMDH-type model has the lowest standard variations of MAPE. This means that traders will be additionally advantaged by reducing risks of making incorrect decisions. In contrast, the ANN model has the largest deviation of MAPE.

The Table 2 shows the values of MAPE for all three prediction models which were built in each of the 13 sessions. The comparison of the prediction performances shows that the ANN model beats the other models in the 8 sessions, whilst the GMDH-type model is superior only in 3 cases. We can see that the ANN predictions are characterised by a high volatility. However in most cases, such predictions are only slightly better than those generated by the GMDH-type model.

\section{Discussion}

We aimed to study Informational Efficiency of the stock market which has been introduced by Fama. This theory has been shown efficient in the cases including financial crises and frauds. The theory is tested by using a model designed for predicting the financial time series.

To achieve the aim we assumed that a prediction model built on retrospective data provides more reliable analysis of the stock market efficiency. This assumption motivated us to analyse Machine Learning methods which are most suitable for building models providing a high prediction performance.

Having discussed our findings we proposed a "deep" learning method for building a high-performance prediction model. The proposed method is based on the Group Method of Data Handling, known as a Deep Learning paradigm capable of building multilayer neural-network models of a near-optimal complexity on given data.

The proposed method have been examined on the Warsaw Stock Exchange data. The comparative results have showed that the developed GMDH-type neural network outperformed the models built by the conventional Machine Learning methods. The complexity of the designed neural-network models was defined as the number of layers and connections between neurons. The models were compared in terms of prediction errors.

It is important to note that GMDH-type ANNs do not require large data sets for learning a prediction model in contrast to the Deep Learning based on a back-propagation method which requires the data augmentation, as shown 
in $[16,22]$. The experimental results shown in the Table 2 provide evidence of this observation.

Observing the experimental results averaged over all 13 sessions shown in the Table 2 we can see that the proposed GMDH-type model provides the predictions whose MAPE is 0.83 , which is below the theoretical threshold 1.0. Thus traders could make a reliable decision that the informational efficiency during the examined period has a weak form.

The alternative AR and ANN models make predictions with a MAPE which is above the threshold. Such prediction models cannot be used in practice.

\section{Conclusion}

Based on the conducted experiments we reported a significantly smaller prediction error of the proposed method than that of the conventional autoregressive and "shallow" neural-network models. This finally allowed us to conclude that traders will be advantaged by the proposed method as follows: (i) the prior knowledge of neural-network model structure is not required as a desired model's structure is learnt from available data and (ii) financial data available for designing prediction models are not required to be large in order to build a reliable prediction model.

\section{Acknowledgements}

The work has been partly supported by the EU Regional Development Fund at the University of Bedfordshire, UK.

\section{Conflict of interest statement}

The authors have declared that no competing interests exist.

\section{References}

1. Nesreen K. Ahmed, Amir F. Atiya, Neamat El Gayar, and Hisham El-Shishiny. An empirical comparison of machine learning models for time series forecasting. Econometric Reviews, 29(5-6):594-621, 2010.

2. Kavous Ardalan. Neurofinance versus the efficient markets hypothesis. Global Finance Journal, 35:170 - 176, 2018.

3. Qing Cao, Karyl B Leggio, and Marc J Schniederjans. A comparison between fama and french's model and artificial neural networks in predicting the chinese stock market. Computers \& Operations Research, 32(10):2499-2512, 2005.

4. Shu-Heng Chen and Chia-Hsuan Yeh. Toward a computable approach to the efficient market hypothesis: An application of genetic programming. Journal of Economic Dynamics and Control, 21(6):1043-1063, June 1997.

5. Arthur Emanuel de Oliveira Carosia, Guilherme Palermo Coelho, and Ana Estela Antunes da Silva. Investment strategies applied to the brazilian stock market: A methodology based on sentiment analysis with deep learning. Expert Systems with Applications, 184:115470, 2021. 
6. Augustas Degutis and Lina Novickytè. The efficient market hypothesis: A critical review of literature and methodology. Ekonomika, 93:7-23, 2014.

7. Eugene Fama. The behavior of stock-market prices. The Journal of Business, 38(1):34105, 1965.

8. Eugene Fama. Efficient capital markets: A review of theory and empirical work. Journal of Finance, 25:383-417, 1970.

9. Stanley J. Farlow. The gmdh algorithm of ivakhnenko. The American Statistician, 35(4):210-215, 1981.

10. Marius-Christian Frunza. Efficient market hypothesis testing. In Marius-Christian Frunza, editor, Solving Modern Crime in Financial Markets, pages 303 - 310. Academic Press, 2016.

11. Kashif Hamid, Muhammad Tahir Suleman, Syed Zulfiqar Ali Shah, and Rana Shahid Imdad Akash. Testing the weak form of efficient market hypothesis: Empirical evidence from asia-pacific markets. Available at SSRN 2912908, 2017.

12. Alexey Ivakhnenko. Polynomial theory of complex systems. IEEE Transactions on Systems, Man and Cybernetics, 4:364-378, 1997.

13. L. Jakaite, V. Schetinin, J. Hladuvka, S. Minaev, A. Ambia, and W. Krzanowski. Deep learning for early detection of pathological changes in x-ray bone microstructures: case of osteoarthritis. Scientific Reports, 11, 2021.

14. Seungwoo Jeon, Bonghee Hong, and Victor Chang. Pattern graph tracking-based stock price prediction using big data. Future Generation Computer Systems, 80:171 - 187, 2018.

15. Takashi Kimoto, Kazuo Asakawa, Morio Yoda, and Masakazu Takeoka. Stock market prediction system with modular neural networks. In Neural Networks, 1990., 1990 IJCNN International Joint Conference on, pages 1-6. IEEE, 1990.

16. Yann LeCun, Yoshua Bengio, and Geoffrey Hinton. Deep learning. Nature, 521(7553):436-444, May 2015.

17. Volodymyr Lytvynenko, Olena Kryvoruchko, Irina Lurie, Nataliia Savina, Oleksandr Naumov, and Mariia Voronenko. Comparative studies of self-organizing algorithms for forecasting economic parameters. International Journal of Modern Education \& Computer Science, 12(6), 2020.

18. H R Madala and A G. Ivakhnenko. Inductive Learning Algorithms for Complex Systems Modeling. CRC Press, Boca Raton, 1994.

19. Burton G Malkiel. The efficient market hypothesis and its critics. Journal of economic perspectives, 17(1):59-82, 2003.

20. E Cruz May, A Bassam, Luis J Ricalde, MA Escalante Soberanis, O Oubram, O May Tzuc, Alma Y Alanis, and A Livas-García. Global sensitivity analysis for a real-time electricity market forecast by a machine learning approach: A case study of mexico. International Journal of Electrical Power \& Energy Systems, 135:107505, 2022.

21. Sidra Mehtab, Jaydip Sen, and Abhishek Dutta. Stock price prediction using machine learning and lstm-based deep learning models. In Symposium on Machine Learning and Metaheuristics Algorithms, and Applications, pages 88-106. Springer, 2020.

22. Francesco Carlo Morabito, Maurizio Campolo, Nadia Mammone, Mario Versaci, Silvana Franceschetti, Fabrizio Tagliavini, Vito Sofia, Daniela Fatuzzo, Antonio Gambardella, Angelo Labate, Laura Mumoli, Giovanbattista Gaspare Tripodi, Sara Gasparini, Vittoria Cianci, Chiara Sueri, Edoardo Ferlazzo, and Umberto Aguglia. Deep learning representation from electroencephalography of early-stage creutzfeldt-jakob disease and features for differentiation from rapidly progressive dementia. International Journal of Neural Systems, 27(02):1650039, 2017. PMID: 27440465.

23. Mahdi Moradi, Mehdi Jabbari Nooghabi, and Mohammad Mahdi Rounaghi. Investigation of fractal market hypothesis and forecasting time series stock returns for tehran stock exchange and london stock exchange. International Journal of Finance 86 Economics, 26(1):662-678, 2021.

24. ANTHONY N MUCCIARDI, FRANCIS J COOK, and JOSEPH NELSON CRAIG. Adaptive learning networks: Development and application in the united states of algorithms related to gmdh. Self-Organizing Methods in Modeling: GMDH Type Algorithms, page $25,2020$. 
25. Qaiser Munir, Kok Sook Ching, Fumitaka Furouka, and Kasim Mansur. The efficient market hypothesis revisited: Evidence from the five small open asean stock markets. The Singapore Economic Review, 57(03):1250021, 2012.

26. J A Müller and F Lemke. Self-Organizing Data Mining: Extracting Knowledge from Data. Trafford Publishing, Canada, 2003.

27. Vasilios Plakandaras, Theophilos Papadimitriou, and Periklis Gogas. Forecasting daily and monthly exchange rates with machine learning techniques. Journal of Forecasting, 34(7):560-573, 2015.

28. V Schetinin, L. Jakaite, and W. Krzanowski. Bayesian averaging over decision tree models: An application for estimating uncertainty in trauma severity scoring. International Journal of Medical Informatics, 112:6 - 14, 2018.

29. V. Schetinin, L. Jakaite, and W. Krzanowski. Bayesian learning of models for estimating uncertainty in alert systems: Application to air traffic conflict avoidance. Integrated Computer-Aided Engineering, 26:1-17, 2018.

30. Vitaly Schetinin and Livija Jakaite. Extraction of features from sleep EEG for Bayesian assessment of brain development. PLOS ONE, 12(3):1-13, 032017.

31. Jürgen Schmidhuber. Deep learning in neural networks: An overview. Neural Networks, $61: 85-117,2015$.

32. Jaydip Sen and T Datta Chaudhuri. Stock price prediction using machine learning and deep learning frameworks. In Proceedings of the 6th International Conference on Business Analytics and Intelligence, Bangalore, India, pages 20-22, 2018.

33. Allan Timmermann and Clive W.J. Granger. Efficient market hypothesis and forecasting. International Journal of Forecasting, 20(1):15 - 27, 2004.

34. Alexandra Gabriela Titan. The efficient market hypothesis: Review of specialized literature and empirical research. Procedia Economics and Finance, 32:442 - 449, 2015. 